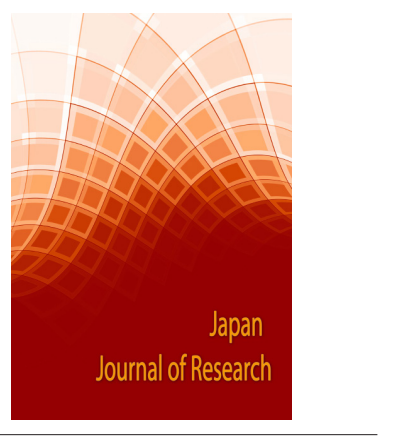

Correspondence

Hanying Feng

Department of Mathematics, Nantong Institute of Technology, Nantong 226002, Jiangsu, P. R. China

E-mail: fhanying@126.com

- Received Date: 03 Apr 2020;

- Accepted Date: 25 Apr 2020;

- Publication Date: 30 Apr 2020.

Keywords

Nonlinear system, Limit cycles, Existence, Uniqueness

\section{Copyright}

(C) 2020 Science Excel. This is an open access article distributed under the terms of the Creative Commons Attribution 4.0 International license.

\title{
Existence and Uniqueness of Limit Cycles for a Class of Nonlinear Systems
}

\author{
Hanying Feng ${ }^{1,2+}$ and ${\mathrm{Zhe} \mathrm{Li}^{2}}^{2}$ \\ 'Department of Mathematics, Nantong Institute of Technology, Nantong 226002, Jiangsu, P. R. China \\ ${ }^{2}$ Department of Mathematics, Shijiazhuang Mechanical Engineering College, Shijiazhuang 050003, Hebei, P. R. China
}

\begin{abstract}
Applying the theory of limit cycles and bifurcation of the plane autonomous system, we study the existence and uniqueness of limit cycles for a class of nonlinear systems with general significance. By using the conclusions obtained, some known results are extended and improved.
\end{abstract}

\section{Introduction}

Hilbert presented the well-known 23 mathematical problems to the Second International Congress of Mathematicians in 1900. The second part of Hilbert's 16th problem deals with polynomial differential equations in the plane. There has been a large body of excellent work on the study of the problems above since 1900 (see, for example, $[1,3,6,7,10,12,13,17]$ and the references cited therein). However, it remains unsolved even for quadratic polynomials. So far, most of the previous important works tackle the quadratic polynomial systems, but there aren't many results dealing with cubic and higher degree polynomial systems.

In this paper, we discuss the number and the distribution of limit cycles for a class of nonlinear systems as following

$$
\begin{aligned}
& d x / d t=-y^{\alpha}(1+n y)^{\beta}+d x^{\alpha}(1+n y)^{\beta} \\
& +l x^{\alpha+1}(1+n y)^{\beta-1}-m(1+n y)^{\gamma} \psi(y), \\
& d y / d t=x^{\alpha}\left[(1+n y)^{\beta}+(a x)^{\beta}\right],
\end{aligned}
$$

where $\alpha, \beta$ is a positive odd integer, $\gamma \geq \alpha+\beta$ and $\psi: R \rightarrow R$. Without loss of generality, we assume $a$ $\leq 0$ (Otherwise, one may make the transformation $\left.x_{1}=-x, t_{1}=-t\right)$.

The paper is organized as the follows. In the next section, by analyzing an equivalent system of (1.1), we discuss the existence and uniqueness of limit cycles of system (1.1). In section 3, by applying the main results in section 2 , we discuss some polynomial differential systems considered earlier by some other authors. Some known results in $[1,3,4,12,17]$ are extended and improved.

\section{The main results and proofs}

In this section, we discuss the existence and uniqueness of limit cycles of system (1.1) by using the qualitative theory of ordinary differential equations.
Noting that $y=-1 / n$ is a straight line isocline, we set $x=x_{1}\left(1+n y_{1}\right), y=y_{1}, d t=\left(1+n y_{1}\right)^{1-\beta} d t_{1}$,

then system (1.1) can be transformed into the following equivalent system (denoting $x_{1}, y_{1}, t_{1}$, again by $x, y, t$, respectively):

$d x / d t=-y^{\alpha}+(1+n y)^{\alpha} x^{\alpha}[d+(l-n) x$

$\left.-a^{\beta} n x^{\beta+1}\right]-m(1+n y)^{\gamma-\beta} \psi(y) \equiv P(x, y)$,

$d y / d t=(1+n y)^{\alpha+1} x^{\alpha}\left(1+(a x)^{\beta}\right) \equiv Q(x, y)$

We now formulate our main results for system (1.1) and (2.1) as follows.

Theorem 2.1. If $a^{\beta} d \alpha(\alpha l+l+n \beta) \leq 0$, then system (1.1) has no limit cycle in the whole plane.

Proof. Take a Dulac's function

$B(y)=(1+n y)^{-\left(\frac{\alpha l+l}{n}\right)}$, we derive that

$\operatorname{div}(B P, B Q)=(1+n y)^{\frac{\alpha n-\alpha l-l}{n}} x^{\alpha-1}\left[\alpha d-a^{\beta}(\alpha l+l+n \beta) x^{\beta+1}\right]$.

Thus, if $a^{\beta} d \alpha(\alpha l+l+n \beta) \leq 0$, then $\operatorname{div}(B P, B Q)$ is of fixed sign in the region $y+\frac{1}{n}>0$ or $y+\frac{1}{n}<0$ and is not identically equal to zero in any subregion since $\beta$ is a positive odd integer. By Dulac's criterion, we know that system (2.1) has no limit cycle in the whole plane. Since system (2.1) is equivalent to system (1.1), we assert that system (1.1) has no limit cycle in the whole plane. This completes the proof.

By Theorem 2.1, we can assume $a^{\beta} d \alpha(\alpha l+l+n \beta)>$ 0 . By the assumption of $a \leq 0$ we have $d \alpha(\alpha l+l+$ $n \beta)<0$. Hence, in the following, we always assume $l>-\frac{n \beta}{\alpha+1}, a<0$ and $d<0$.

Lemma 2.2. If system (2.1) has limit cycles, then they must intersect the line $l_{1}: x=-\frac{\alpha d}{\alpha l+l+n \beta}$

Proof. Take a Dulac's function $B(y)=(1+n y)^{\beta}$, we have 
$\operatorname{div}(B P, B Q)=(1+n y)^{\alpha+\beta} x^{\alpha-1}[\alpha d+(\alpha l+l+n \beta) x]$.

Hence, if system (2.2) has limit cycles, then they must intersect the line $x=-\frac{\alpha d}{\alpha l+l+n \beta}$

Lemma 2.3. If system (2.1) has limit cycles around the origin, then they must not intersect the line $l_{2}: x=-\frac{1}{a}$.

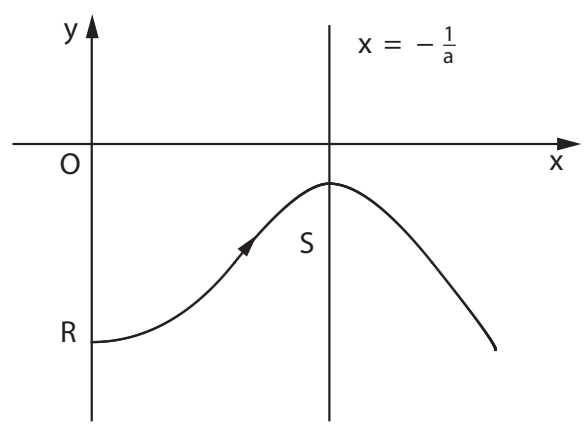

Proof. Let $\Gamma$ be a limit cycle around the origin which intersects the $y$-axis at $R$ and the line $x=-\frac{1}{a} \quad$ on $S$. Since $Q(x, y)=(1+n y)^{\alpha+1} x^{\alpha}\left(1+(a x)^{\beta}\right)>0$ between the region $x=0$ and $x=-\frac{1}{a}$; $Q(x, y)<0$ on the right hand of the line $x=-\frac{1}{a}, \Gamma$ will go in a right downward direction after it passes through the point $S$. Moreover, it is impossible for it to go around the origin. This contradicts the assumption that $\Gamma$ is a limit cycle around the origin. The proof is complete.

Theorem 2.4. If $a d \geq l+\frac{l}{\alpha}+\frac{n \beta}{\alpha}$, then system (2.1) has no limit cycle around the origin.

Proof. By Lemma 2.2, if system(2.1) has a limit cycle around the origin, it must intersect the line $x=-\frac{\alpha d}{\alpha l+l+n \beta}$. By Lemma 2.3, it must not intersect the line $x=-\frac{1}{a}$. Hence the line $x=-\frac{\alpha d}{\alpha l+l+n \beta}$ must lie in the left hand of the line $x=-\frac{1}{a}$, so we have $-\frac{\alpha d}{\alpha l+l+n \beta}<-\frac{1}{a}$, i.e., $a d<l+\frac{l}{\alpha}+\frac{n \beta}{\alpha}$. Therefore, if $a d \geq l+\frac{l}{\alpha}+\frac{n \beta}{\alpha}$, hen system (2.1) has no limit cycle around the origin. The proof is complete.

From what has been discussed above, we can assume $0<a d<l+\frac{l}{\alpha}+\frac{n \beta}{\alpha}$. As follow we will prove the existence and uniqueness of limit cycles. Besides we know that if system (2.1) has a limit cycle around the origin, it must lie in the region:

$$
D=\left\{(x, y) \mid x<-\frac{1}{a}, y>-\frac{1}{n}\right\} .
$$

Let

$$
x_{2}=x, e^{-y_{2}}=1+n y, d t_{2}=-(1+n y)^{\alpha} d t .
$$

system (2.1) becomes

$$
\begin{aligned}
& d x / d t=-\varphi(y)-F(x), \\
& d y / d t=g(x),
\end{aligned}
$$

where

$$
\begin{gathered}
\varphi(y)=\left(\frac{e^{y}-1}{n}\right)^{\alpha}-m e^{-y(\gamma-\alpha-\beta)} \psi\left(\frac{e^{-y}-1}{n}\right), \\
F(x)=x^{\alpha}\left[d+(l-n) x-a^{\beta} n x^{\beta+1}\right], \\
g(x)=n x^{\alpha}\left(1+(a x)^{\beta}\right) .
\end{gathered}
$$

Denote

$$
\begin{gathered}
f(x)=F^{\prime}(x)=x^{\alpha-1}\left[\alpha d+(\alpha+1)(l-n) x-a^{\beta} n(\alpha+\beta+1) x^{\beta+1}\right], \\
G(x)=\int_{0}^{x} g(x) d x=\frac{n}{\alpha+1} x^{\alpha+1}+\frac{a^{\beta} n}{\alpha+\beta+1} x^{\alpha+\beta+1} .
\end{gathered}
$$

Lemma 2.5. If $d<0,|d|<<1$, then the singular point $O$ of system (2.1) is a stable focus; if $d=0$ then the singular point $O$ is an unstable focus.

Proof. Let $z=G(x)$, as $x \rightarrow 0, G(x) \sim \frac{n}{\alpha+1} x^{\alpha+1}$. We denote by $x_{1}(z)$ $>0$ the inverse function of $G(x)$ in the interval $\left(0,-\frac{1}{a}\right)$, and $x_{2}(z)$ $>0$ by the inverse function of $G(x)$ in the half plane $x<0$, as $z \rightarrow 0$, $x_{1}(z) \sim\left(\frac{\alpha+1}{n}\right)^{\frac{1}{\alpha+1}} z^{\frac{1}{\alpha+1}}, \quad x_{2}(z)=-x_{1}(z)$. Denote $F_{i}(z)=F\left(x_{i}(z)\right), \quad i=1,2$. It is easy to verify that $\varphi(y)$ and $F_{i}(z)$ satisfy all the conditions of Corollary 4 in [10] for $d \leq 0$ and $a<0$.

As $z \rightarrow 0, F_{1}(z)-F_{2}(z)=2 d\left(\frac{\alpha+1}{n} z\right)^{\frac{\alpha}{\alpha+1}}-2 a^{\beta}\left(\frac{\alpha+1}{n} z\right)^{\frac{\alpha+\beta+1}{\alpha+1}}$, it is easy to see that for $0<z<<1$, if $d<0$, if $d=0, F_{1}(z)-F_{2}(z)>0$. Hence, if $d<0$, then the origin $O$ is an unstable focus of system (2.3); if $d=0$; then the origin $O$ is a stable focus. Noticing the transformation (2.2), it follows that if $d$ $<0$, then the origin $O$ is a stable focus of system (2.1); if $d=0$; then the origin $O$ is an unstable focus. The proof is complete.

Theorem 2.6. If $0<a d<l+\frac{l}{\alpha}+\frac{n \beta}{\alpha},|d| \ll 1$, then system (1.1) has limit cycles around the origin.

Proof. By Lemma 2.5 and the Hopf's bifurcation Theorem in [16], the conclusion of Theorem 2.6 can be obtained directly. This completes the proof.

Theorem 2.7. If $0<a d<l+\frac{l}{\alpha}+\frac{n \beta}{\alpha}, n, m>0, \psi(x) \geq 0$ with $\psi(0)=0$ and

$$
\psi^{\prime}(x)>\frac{-\alpha x^{\alpha-1}}{m(1+n x)^{\gamma-\beta+1}}, \text { for } x \in\left(-\frac{1}{n},+\infty\right),
$$

then system (1.1) has at most one limit cycle around the origin, and if it exists, it must be unstable.

Proof. Noticing the transform (2.2), the origin $O$ doesn't change, and $y>-\frac{1}{n}$ becomes $y \in(-\infty,+\infty)$, so we will discuss the limit cycles of system (2.3) in the region $D=\left\{(x, y) \mid x<-\frac{1}{a}\right\}$. Consider system (2.3), it is easy to determine $\varphi(0)=0$ and $x g(x)>0$ for $x<-\frac{1}{a}$ and $x \neq 0$. At the same time, we claim that $\varphi^{\prime}(y)>0$. In fact, by (2.4), one has

$\varphi^{\prime}(y)=\frac{\alpha}{n} e^{y}\left(\frac{e^{y}-1}{n}\right)^{\alpha-1}+m(\gamma-\alpha-\beta) e^{-y(\gamma-\alpha-\beta)} \psi\left(\frac{e^{-y}-1}{n}\right)+\frac{m}{n} e^{-y(\gamma-\alpha-\beta+1)} \psi^{\prime}\left(\frac{e^{-y}-1}{n}\right)$ 


$$
\begin{aligned}
& >\frac{\alpha}{n} e^{y}\left(\frac{e^{y}-1}{n}\right)^{\alpha-1}+m(\gamma-\alpha-\beta) e^{-y(\gamma-\alpha-\beta)} \psi\left(\frac{e^{-y}-1}{n}\right) \\
& +\frac{m}{n} e^{-y(\gamma-\alpha-\beta+1)} \frac{-\alpha\left(\frac{e^{-y}-1}{n}\right)^{\alpha-1}}{m e^{-y(\gamma-\beta+1)}} \\
& =m(\gamma-\alpha-\beta) e^{-y(\gamma-\alpha-\beta)} \psi\left(\frac{e^{-y}-1}{n}\right) \\
& >0 .
\end{aligned}
$$

Again,

$$
\left(\frac{f(x)}{g(x)}\right)^{\prime}=-\frac{1}{n^{2} x^{2}\left(1+(a x)^{\beta}\right)^{2}}\left[\alpha d n+a^{\beta} \alpha d n(\beta+1) x^{\beta}+a^{\beta} \beta n(\alpha l+l+n \beta) x^{\beta+1}\right] .
$$

Let

$$
h(x)=\alpha d n+a^{\beta} \alpha d n(\beta+1) x^{\beta}+a^{\beta} \beta n(\alpha l+l+n \beta) x^{\beta+1}
$$

then it follows

$$
h^{\prime}(x)=a^{\beta} \alpha d n(\beta+1) \beta x^{\beta-1}+a^{\beta} \beta n(\beta+1)(\alpha l+l+n \beta) x^{\beta} .
$$

It is easy to verify that $h(x)$ has a unique point, namely $x_{0}=-\frac{\alpha d}{\alpha l+l+n \beta}$ at which $h(x)$ attains its maximum, and $h\left(x_{0}\right)=\alpha d n\left[1-\left(\frac{a \alpha d}{\alpha l+l+n \beta}\right)^{\beta}\right]$. Noticing that $0<a d<l+\frac{l}{\alpha}+\frac{n \beta}{\alpha}$, we know that $h\left(x_{0}\right)<0, h(x) \quad 0$. Therefore, it follows that $\left(\frac{f(x)}{g(x)}\right)^{\prime}>0$.

By Lemma 2.5, if $d<0$ then the origin $O$ is a stable focus of system (1.1). Applying the Zhang Zhifen's Theorem in [18], we have that system (1.1) has at most one limit cycle around the origin, and if it exists, it must be unstable. This completes the proof.

\section{The applications of the main results}

In [3], the author considered the system

$$
\begin{aligned}
& d x / d t=-y^{\alpha}(1+n y)^{\alpha}+d x^{\alpha}(1+n y)^{\alpha}+l x^{\alpha+1}(1+n y)^{\alpha-1}-m y^{\alpha+1}(1+n y)^{\alpha}, \\
& d y / d t=x^{\alpha}\left[(1+n y)^{\alpha}+(a x)^{\alpha}\right],(0 \leq m \leq n),
\end{aligned}
$$

which is a special case of (1.1) when $\beta=\gamma=\alpha, \psi(y)=y^{\alpha+1}$ in system (1.1). Now we verify that $\psi(y)=y^{\alpha+1}$ satisfies (2.4) in Theorem 2.7. Let $\mathrm{T}(y)=$ $y(1+n y)$. It is easy to know that $\mathrm{T}(y)$ has a minimal value $T\left(y_{0}\right)=-\frac{1}{4 n}$ at $y_{0}=-\frac{1}{2 n}$. Noticing that $0<m \leq n$, we can have that $-\frac{1}{4 n}>-\frac{\alpha}{m(\alpha+1)}$. It follows that $y(1+n y) \geq-\frac{1}{4 n}>-\frac{\alpha}{m(\alpha+1)}$, then $(\alpha+1) y^{\alpha}>-\frac{\alpha y^{\alpha-1}}{m(1+n y)}$. Therefore $\psi^{\prime}(y)=(\alpha+1) y^{\alpha}>-\frac{\alpha y^{\alpha-1}}{m(1+n y)}$, that is, (2.4) holds. Thus the following corollaries can be derived by Theorems 2.1, 2.4, 2.6, 2.7 .

Corollary 3.1. For system (3.1), if $\operatorname{ad}[l(\alpha+1)+n \alpha] \leq 0$, then there is no limit cycle in the whole plane; if $a d \geq[l(\alpha+1)+n \alpha] / \alpha$, then there is no limit cycle around the origin.

Corollary 3.2. For system (3.1), we have the following conclusions:

(i). If $0<a d<[l(\alpha+1)+n \alpha] / \alpha$, then there is at most one limit cycle around the origin, and if it exists, it must be unstable;

(ii). If $0<a d<[l(\alpha+1)+n \alpha] / \alpha$ and $|d|<<1$, then there is a unique unstable limit cycle around the origin.

In [12], the author discussed the system

$$
\begin{aligned}
& d x / d t=-y+d x+x^{2}+d x y-(m+1) y^{2}-m y^{3}, \\
& d y / d t=x(1+a x+y),(0 \leq m \leq 1),
\end{aligned}
$$

which is a special case of (3.1) when $\alpha=1, l=1, n=1$ in system (3.1) or a special case of when $\alpha=\beta=\gamma=1, l=1, n=1, \psi(y)=y^{2}$ in system (1.1). Thus we have

Corollary 3.3. For system (3.2), if ad $\leq 0$ then there is no limit cycle in the whole plane;

If ad $\geq 3$, then there is no limit cycle around the origin.

Corollary 3.4. For system (3.2), we have the following conclusions:

(i). If $0<a d<3$, then there is at most one limit cycle around the origin, and if it exists, it must be unstable;

(ii). If $0<a d<3$ and $|d|<1$, then there is a unique unstable limit cycle around the origin.

In [17], the author discussed the quadratic system

$$
\begin{aligned}
& d x / d t=-y+d x+x^{2}+d x y-y^{2} \\
& d y / d t=x(1+a x+y),
\end{aligned}
$$

which is a special case of (3.2) when $\mathrm{m}=0$ which is a special case of (3.2) when $m=0$ in system (3.2) or a special case of (1.1) when $\alpha=1, \beta$ $=1,1=1, \mathrm{n}=1, \mathrm{~m}=0$ in system (1.1). From what has been discussed above, it is easy to see that above Corollaries 3.3, 3.4 still hold for system (3.3).

Remark 3.5. Corollaries 3.3, 3.4 not only contain all results in [11] and [17], but also show sufficient conditions for the existence of limit cycles which was not involved in [17].

In [4], the authors studied the system

$$
\begin{aligned}
& d x / d t=-y(1-y)^{\alpha}-d x(1-y)^{\alpha}+l x^{2}(1-y)^{\alpha-1}, \\
& d y / d t=x\left[(1-y)^{\alpha}+(a x)^{\alpha}\right] .
\end{aligned}
$$

On substituting

$$
\hat{x}=x, \quad \hat{y}=-y, \quad \hat{t}=-t
$$

into (3.4) and dropping the hats for ease of notation, system (3.4) reduces to the equivalent system.

$$
\begin{aligned}
& d x / d t=-y(1+y)^{\alpha}+d x(1+y)^{\alpha}-l x^{2}(1+y)^{\alpha-1}, \\
& d y / d t=x\left[(1+y)^{\alpha}+(a x)^{\alpha}\right],
\end{aligned}
$$

which is a special case of (1.1) when $m=0, \alpha=1, \beta=\alpha, n=1$ in system (1.1). Noticing the negative sign before $l$ in system (3.6) and the transformation (3.5), by Theorems 2.1, 2.4, 2.6, 2.7, we have the following results.

Corollary 3.6. If ad $(\alpha-2 l) \leq 0$, then system (3.6) has no limit cycle in the whole plane; If $a d \geq \alpha-2 l$; then system (3.6) has no limit cycle around the origin.

Corollary 3.7. For system (3.6), we have the following conclusions:

(i). If $0<a d<\alpha-2 l$, then system (3.6) has at most one limit cycle around the origin, and if it exists, it must be stable; 
(ii). If $0<a d<\alpha-2 l$ and $|d|<<1$ then system (3.6) has a unique stable limit cycle around the origin.

Remark 3.8. Corollaries 3.6, 3.7 contain the main results in [4].

In [1], the author considered the following quadratic system with a degenerate critical point:

$$
\begin{aligned}
& d x / d t=-y-d x+l x^{2}+d x y+y^{2}, \\
& d y / d t=x(1+a x-y),
\end{aligned}
$$

which is a special case of (3.4) when $\alpha=1$ in system (3.4) or a special case of (1.1) when $\alpha=\beta=1$ and $m=0$ in system (1.1). From what has been discussed above, it is easy to see that above Corollaries 3.6, 3.7 in which when $\alpha=1$ still hold for system (3.7).

In [13], the authors studied the system

$$
\begin{aligned}
& d x / d t=-y^{\alpha}(1+y)+d x^{\alpha}(1+y)+x^{\alpha+1}, \\
& d y / d t=x^{\alpha}(1+a x+y),
\end{aligned}
$$

which is a special case of (1.1) when $m=1, l=1, \beta=1, n=1$ in system (1.1). It is easy to derive the following corollaries by Theorems 2.1, 2.4, 2.6, 2.7 .

Corollary 3.9. For system (3.8), if ad $\leq 0$, then there is no limit cycle in the whole plane; if $a d \geq \frac{\alpha+2}{\alpha}$, then there is no limit cycle around the origin.

Corollary 3.10. For system (3.8), we have the following conclusions:

(i). If $0<a d<\frac{\alpha+2}{\alpha}$, then there is at most one limit cycle around the origin, and if it exists, it must be unstable;

(ii). If $0<a d<\frac{\alpha+2}{\alpha}$ and $|d|<1$, then there is a unique unstable limit cycle around the origin.

\section{Funding Information}

Supported by NNSF of China (11371368) and NITNSF (201824)

\section{References}

1. Coppel WA. Qudratic system with a degenerate critical point. Bull Aust Math Soc. $1998 ; 38(1): 1-10$.
2. Cheng Z, Lin Y, Cao J. Dynamical behaviors of a partial-dependent predatorprey system. Chaos, Solitons \& Fractals. 2006; 28(1):67-75.

3. Feng H. Limit cycles for a class of polynomial systems and applications. Electronic Journal of Differential Equations. 2003; 2003(105):1-7.

4. Feng H, Discussion and application on the limit cycles for a class of higher polynomial system, J. Ordn. Eng. Coll., (6)1994, 223-231 (in Chinese).

5. Gasullb A, Llibreb J. Uniqueness of limit cycles for a class of Lienard systems with applications. J Math Anal Appl. 1989; 141:442-450.

6. Guo C, Quan S. Limit cycles of a class of higher-degree polynomial systems and their application. (Chinese) J Lanzhou Univ Nat Sci. 1997; 33(3): 9-13.

7. Giné J, Llibre J. Integrability, degenerate centers, and limit cycles for a class of polynomial differential systems. Comput Math Appl. 2006; 56: 1453-1462.

8. Hwang C, Hsieh, R. Lin, A linear continuous feedback control of Chua's circuit. Chaos, Solitons \& Fractals, (8)1997, 1507-1515.

9. Hirano N, Rybicki S. Existence of limit cycles for coupled van der Pol equations. J Diff Eqs. 2003; 195: 194-209.

10. Han M. Several criteria for the existence of periodic solutions of autonomous system in the plane. J Nanjing Univ. 1985; 21: 233-244 (in Chinese).

11. Huang $\mathrm{X}$, Zhu L, Cheng A. Limit cycles in a general two-stroke oscillation. Nonlinear Anal. 2006; 64: 22-32.

12. Liu D. Discussion of the limit cycles for system $d x / d t=-y+d x+x^{2}+d x y-$ $(\alpha+1) y^{2}-\alpha y^{3}, d y / d t=x(1+\alpha x+y),(0 \leq \alpha \leq 1)$. J Math. 1989; 9: 367-373 (in Chinese).

13. Liu D. Existence and uniqueness of limit cycle for a class of polynomial system. J Shenyang Normal Univ. 1999; 1:1-6 (in Chinese).

14. Ohta $\mathrm{H}$, Ueda Y. Blue sky bifurcations caused by unstable limit cycle leading to voltage collapse in an electric power system. Chaos, Solitons \& Fractals, $2002 ; 14: 1227-1237$

15. Sabatini M. Existence and uniqueness of limit cycles in a class of second order ODEs with inseparable mixed terms. Chaos, Solitons \& Fractals. 2010; 43: $25-30$

16. Zhang J. Geometrical theory and bifurcation problems of ordinary differential equations, Bejing: Peking University Press, 1987. (second edition)

17. Zou Y. The limit cycles of the equations $d x / d t=-y+d x+b x^{2}+b d x y-b y^{2}$ $d y / d t=x(1+\alpha x+y)$. J Wuhan Univ. 1977; 4: 7-9 (in Chinese).

18. Zhang Z, Ding T, Huang W, et al. Qualitative theory of differential equations, Beijing: Science Press, 1985. 